Urologe 2013 · 52:1515-1516

DOI 10.1007/s00120-013-3255-9

Online publiziert: 25. Oktober 2013

(c) Springer-Verlag Berlin Heidelberg 2013

J. Gschwend

Urologische Klinik und Poliklinik, Klinikum rechts der Isar, Technische Universität München

\title{
Rationale medikamentöse Tumortherapie
}

mit den Nachbardisziplinen koordinieren. Genau darin liegen die Kraft und die Kompetenz der Urologie.

Bei Betrachtung der einzelnen Tumorentitäten sticht in den vergangenen 2 Jahren das kastrationsrefraktäre, metastasierte Prostatakarzinom heraus. War in den letzten 10 Jahren nur die Docetaxel-basierte Chemotherapie in diesem Erkrankungsstadium verfügbar, so eröffneten sich mit Abirateron, Enzalutamid, Cabazitaxel und Alpharadin vier neue Optionen, die alle mit einem Überlebensvorteil für den Patienten verbunden sind. Der rationale Einsatz dieser Medikamente basierend auf den zugelassenen Indikationen beantwortet aber nicht zwangsläufig die Frage nach deren optimaler Sequenz oder gar Kombination. Hier werden Daten aus weiteren klinischen Studien oder Daten aus der Versorgungsforschung dringlich erwartet.

Diese Situation wird von der Entwicklung der medikamentösen Therapieoptionen beim metastasierten Nierenzellkarzinom widergespiegelt. Nach der Zulassung der ersten Tyrosinkinaseinhibitoren (TKI) Sunitinib und Sorafenib 2006 wurde eine Vielzahl von Studien zur Erstlinien- und Zweitlinientherapie durchgeführt und publiziert. Mittlerweile sind für das Nierenzellkarzinom 6 mehr oder weniger vergleichbare Wirkstoffe zugelassen, die je nach Zulassungstext für unterschiedliche klinische Situationen eingesetzt werden können. Da Kombinationen aufgrund der Toxizität keine Rolle spielen, ist hier besonders die Frage der idealen Sequenz gestellt, so es diese denn überhaupt gibt. Zwei deutsche Studienprotokolle (Switch I und II) untersuchen aktuell die sequentielle Anwendung von je zwei
TKI und werden wichtige Fragen beantworten können, die die Versorgungsrealität stellt.

\section{》) Wichtig sind der optimale Einsatz der verfügbaren Substanzen in den jeweiligen Stadien sowie das richtige Timing der Therapie}

Beim fortgeschrittenen oder metastasierten Urothelkarzinom der Harnblase sind auch in den letzten Jahren leider wenig substantielle Fortschritte zu verzeichnen gewesen. Einzig die Zulassung von Vinflunine in der Zweitlinientherapie hat etwas frischen Wind in die Therapielandschaft gebracht. Umso wichtiger sind die Frage des optimalen Einsatzes der verfügbaren wirksamen Substanzen in den jeweiligen Stadien sowie insbesondere das richtige Timing der Therapie. Der perioperativen Chemotherapie im neoadjuvanten, induktiven oder adjuvanten Setting widmet sich ein Beitrag in diesem Heft ebenso intensiv wie ein zweiter der sich mit der Frage der rationalen Systemtherapie des bereits metastasierten Blasenkarzinoms auseinandersetzt.

Die eher seltenen, aber aufgrund des oft geringen Alters der Patienten besonders bedeutsamen Keimzelltumoren verdienen ebenso eine aktuelle Standortbestimmung. Bei Heilungsraten von fast $90 \%$ über alle Stadien bedarf es einer optimalen Therapiesteuerung bei gleichzeitig kritischer Beachtung der Belastung betroffener Patienten durch Toxizität und einem nicht unerheblichen Zweitmalignomrisiko. Eine stadienorientierte $\mathrm{Re}$ duktion der Therapieintensität und damit ventionen oder radioonkologische Maßnahmen im interdisziplinären Konzert 
der Nebenwirkungen ist Gegenstand aktueller Überlegungen in den internationalen und nationalen Hodentumorgruppen.

Schließlich soll auch das eher seltene und dadurch leider eher wenig beachtete Plattenepithelkarzinom des Penis in den Fokus gerückt werden. Bei einer insgesamt eher dürftigen Datenlage zur Therapie des lokal fortgeschrittenen oder lymphogen metastasierten Peniskarzinoms werden die verfügbaren Informationen zu multimodalen Therapiekonzepten analysiert und mögliche Szenarien für die $\mathrm{Zu}$ kunft entwickelt.

Nicht zuletzt kommt der rationalen Bildgebung zur Verlaufskontrolle unter systemischer Therapie eine entscheidende Bedeutung bei. Da auch in den verfügbaren Leitlinien meist keine evidenzbasierten Empfehlungen ausgesprochen werden, versucht dieser Beitrag eine wissenschaftlich bestmögliche Strategie zur Indikation und Durchführung bildgebender Diagnostik zu entwickeln.

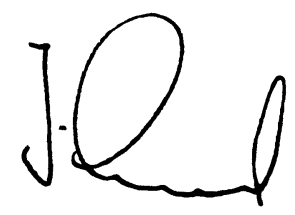

J. Gschwend

\section{Korrespondenzadresse}

Prof. Dr. J. Gschwend
Urologische Klinik und
Poliklinik, Klinikum rechts der
Isar, Technische Universität
München
Ismaninger Straße 22,
81675 München
juergen.gschwend@lrz.tum.de

\section{Einhaltung ethischer Richtlinien}

Interessenkonflikt. J. Gschwend gibt an, dass kein Interessenkonflikt besteht.

\section{Unterstützung klinischer Studien und anderer wissen- schaftlicher Untersuchungen}

Klinische Studien und präklinische Untersuchungen sind unerlässlich, um neue Therapien und Diagnostika zu erproben und die Behandlung unserer Patienten nachhaltig zu verbessern. Erfreulicherweise steigt die Zahl klinischer Studien an und hierbei werden die Pathologie und die gewebebasierte Forschung vermehrt einbezogen. Die Deutsche Gesellschaft für Pathologie e.V. (DGP) und die durch sie vertretenen Pathologen wollen und können zu diesem Prozess als gleichwertige Partner beitragen und unsere klinischen Partner dabei bestmöglich unterstützen. Mit unseren Technologien und unserer spezifischen Expertise können wir zur Qualität und zum Gelingen präklinischer Untersuchungen, klinischer Studien und der Implementierung der Ergebnisse in der klinisch-pathologischen Diagnostik beitragen. Wir wollen Forschung in und mit der Pathologie fördern und uns aktiv an der Gestaltung von Forschung beteiligen. Die DGP hat ein großes Interesse an (guter) klinischer Forschung und unterstützt diese nachdrücklich. Vor dem Hintergrund immer komplexer werdender Anforderungen und Interessenlagen stellt die DGP einen sachlich begründeten und strukturierten Kriterienkatalog vor, der für die Leiter und Organisatoren klinischer Studien und Pathologen gleichermaßen als Informationsquelle und Entscheidungshilfe bei Studien und anderen wissenschaftlichen Untersuchungen dienen soll. Die von der DGP erarbeitete Stellungnahme zur Beteiligung und Unterstützung klinischer Studien und anderer wissenschaftlicher Untersuchungen (Der Pathologe 2013;34:466-475) beinhaltet eine Checkliste für die Planung klinischer Studien mit und an Gewebeproben und stellt einen Fragebogen vor, der als Vorlage für Materialanforderungen dienen kann. Beide berücksichtigen allgemeine Aspekte (Studieninitiator, Studienfinanzierung, Studienart, Studienziel, Art und Menge des angefragten Materials), rechtliche Grundlagen (Einverständniserklärung des Patienten, Sachwalterschaft für Studiengut, Verbleib des Studienmaterials), Datenschutzbestimmungen, Ethikvotum, Fachstandards (z.B. Studienpathologie ist Facharzt für Pathologie, Befund- und Materialarchivierung) und Aufwandsentschädigungen. Eine kritische Ausei- nandersetzung mit der Stellungnahme durch andere medizinische Fachgesellschaften und Organisationen ist erwünscht und soll zur nachhaltigen Verbesserung der Forschung mit und an humanem Gewebe beitragen.

Im Auftrag der Deutschen Gesellschaft für Pathologie e.V.: Christoph Röcken, Heinz Höfler, Michael Hummel, Richard Meyermann, Christian Zietz, Peter Schirmacher

Korrespondenzadresse:

Prof. Dr. med. Christoph Röcken Institut für Pathologie

Christian-Albrechts-Universität

Arnold-Heller-Str. 3, Haus 14

24105 Kiel

Tel: +49(0)431-597-3401

Fax: +49(0)431-597-3462

E-Mail: christoph.roecken@uksh.de www.dgp-berlin.de 